\title{
The continuous cohomology of period domains over local fields
}

\author{
Sascha Orlik
}

\begin{abstract}
In this paper we consider period domains over local fields for quasi-split reductive groups. We compute the continuous $\ell$-adic cohomology with compact support of them in the case of a basic isocrystal. This paper is a continuation of [O2] where we considered the étale cohomology with torsion coefficients of these spaces.
\end{abstract}

Mathematics Subject Classification (2000). Primary 14L05; Secondary 14G22, 14L24.

Keywords. Period domain, reductive group, isocrystal, étale cohomology.

\section{Introduction}

This paper deals with the computation of the continuous $\ell$-adic cohomology with compact support of period domains over local fields. We consider the case where the underlying reductive group is quasi-split and the isocrystal is basic. In [O2] we computed the étale cohomology with torsion coefficients of them. If we pass to the usual $\ell$-adic cohomology defined by the projective limit of the cohomology groups with values in $\mathbb{Z} / \ell^{n} \mathbb{Z}$, we get continuous generalized Steinberg representations in Banach spaces. This was first pointed out by R. Huber in the Drinfeld case (compare [H2], Example 2.7). In order to get smooth representations with values in $\mathbb{Q}_{\ell}$, he observes that one rather has to consider the continuous $\ell$-adic cohomology with compact support of these objects. The latter cohomology theory was implicitly introduced by him for adic spaces [H2] (except that he does not tensor the cohomology with $\mathbb{Q}_{\ell}$ ). In the case of schemes this construction goes back to Jannsen [J].

We briefly recall the notion of a period domain for reductive groups [RZ]. We refer to the next section for a more detailed description. Let $L$ be an algebraically closed field of characteristic $p>0$. Denote by $K_{0}=W(L)_{\mathbb{Q}}$ the fraction field of the ring of Witt vectors $W(L)$ and by $\sigma$ the Frobenius automorphism of $K_{0}$. We consider a triple

$$
(G, b,\{\mu\})
$$

consisting of a quasi-split reductive group $G$ defined over $\mathbb{Q}_{p}$, an element $b \in G\left(K_{0}\right)$ and a conjugacy class $\{\mu\}$ of one-parameter subgroups of $G$. According to Kottwitz 
[K1], the element $b$ gives rise to an isocrystal with $G$-structure $N_{b}$ on $L$. By definition this is an exact faithful tensor functor

$$
N_{b}: \operatorname{Rep}_{\mathbb{Q}_{p}}(G) \rightarrow \operatorname{Isoc}(L)
$$

from the category of finite-dimensional rational $G$-representations into the category of isocrystals over $L$. Here an isocrystal over $L$ is a pair $(N, \Phi)$ consisting of a finitedimensional $K_{0}$-vector space $N$ and a $\sigma$-linear bijective endomorphism $\Phi$ of $N$. To the conjugacy class $\{\mu\}$ is associated a flag variety $\mathscr{F}=\mathscr{F}(G,\{\mu\})$ defined over some finite extension $E$ of $\mathbb{Q}_{p}$. Let $\mathscr{F}^{\text {rig }}$ be the rigid-analytic variety attached to $\mathscr{F}$. By choosing a $G$-representation $V$, we get for each point $x \in \mathscr{F}^{\text {rig }}$ a filtered isocrystal $\left(N_{b}(V), \mathcal{F}_{x}\right)$ over some field extension $K$ of $K_{0}$. A filtered isocrystal $(N, \Phi, \mathcal{F})$ is called weakly admissible (in the sense of Fontaine [Fo]) if

$$
\sum_{i} i \cdot \operatorname{dim} g r_{\mathcal{F}^{\prime}}^{i}\left(N^{\prime} \otimes_{K_{0}} K\right) \leq \operatorname{ord}_{p} \operatorname{det}\left(\Phi^{\prime}\right)
$$

for all subisocrystals $\left(N^{\prime}, \Phi^{\prime}\right)$ of $(N, \Phi)$ and equality for $\left(N^{\prime}, \Phi^{\prime}\right)=(N, \Phi)$. Here $\mathcal{F}^{\prime}$ denotes the filtration on $N^{\prime} \otimes_{K_{0}} K$ induced by $\mathcal{F}$. In [RZ] it is shown that the points $x \in \mathscr{F}^{\text {rig }}$ which yield a weakly admissible filtered isocrystal $\left(N_{b}(V), \mathcal{F}_{x}\right)$ for every representation $V$ form an admissible open subset $\mathscr{F}_{b}^{\text {wa }}$ of $\mathscr{F}^{\text {rig }}$. It has therefore the structure of a rigid-analytic variety over $K_{0}$. It is defined over the composite $E_{s}=E \cdot \mathbb{Q}_{p}$, where $\mathbb{Q}_{p}$ is the field of definition of $N_{b}$ (the unramified extension of degree $s$ of $\mathbb{Q}_{p}$ in $K_{0}$ ). The space $\mathcal{F}_{b}^{\text {wa }}$ is called the period domain with respect to $(G, b,\{\mu\})$. In this paper we consider the case of a basic isocrystal. This means that the automorphism group $J$ of $N_{b}$ is an inner form of $G$. In general it is an inner form of some Levi subgroup of $G$.

Of general interest is the computation of the étale cohomology of period domains. The cohomology groups are equipped in a natural way with actions of the $p$-adic Lie group $J\left(\mathbb{Q}_{p}\right)$ and the absolute Galois group $\Gamma_{E_{s}}=\operatorname{Gal}\left(\bar{E}_{s} / E_{s}\right)$. In this paper we compute the continuous $\ell$-adic cohomology with compact support of $\mathcal{F}_{b}^{\text {wa }}$, where $\ell \in \mathbb{N}$ is a prime number with $(\ell, p)=1$. The computation proceeds as follows. Consider the adic spaces $\left(\mathcal{F}_{b}^{\text {wa }}\right)^{\text {ad }}, \mathcal{F}^{\text {ad }}$ associated to $\mathscr{F}_{b}^{\text {wa }}$ and $\mathscr{F}$. Let $Y^{\text {ad }}$ be the closed complement of $\left(\mathscr{F}_{b}^{\text {wa }}\right)^{\text {ad }}$ in $\mathscr{F}^{\text {ad }}$. In [O2] we constructed an acyclic resolution of the constant étale sheaf $\mathbb{Z} / n \mathbb{Z}$ on $Y^{\text {ad }}$ in terms of the Tits building of $J$. The resulting spectral sequence degenerates in $E_{2}$ and computes the cohomology of $Y^{\text {ad }}$ with coefficients in $\mathbb{Z} / n \mathbb{Z}$. In this paper we take up this idea and generalize this construction to an acyclic resolution of a certain object in the derived category of étale sheaves on $Y^{\text {ad }}$. The hypercohomology of this object appears in a long exact cohomology sequence next to the continuous $\ell$-adic cohomology groups of $\mathscr{F}_{b}^{\text {wa }}$ and of $\mathcal{F}$. As in loc. cit. we get a spectral sequence which computes the hypercohomology of this object and hence the cohomology of $\mathcal{F}_{b}^{\text {wa }}$. The cohomology groups are computed as objects in the category of smooth $J\left(\mathbb{Q}_{p}\right) \times \Gamma_{E_{s}}$-representations. Our 
result is the following:

$$
H_{c}^{*}\left(\mathcal{F}_{b}^{\mathrm{wa}}, \mathbb{Q}_{\ell}\right)=\bigoplus_{[w] \in W^{\mu} / \Gamma_{E_{s}}} v_{P_{[[w]}}^{J} \otimes \operatorname{ind}_{[w]}\{-l([w])\}\left[-\left|\Delta \backslash I_{[w]}\right|\right] .
$$

Here $W^{\mu}$ denotes the set of Kostant representatives in the Weyl group $W$ of $G$ with respect to the stabilizer of $\mu$. The index set $W^{\mu} / \Gamma_{E_{s}}$ consists of the orbits for the natural action of $\Gamma_{E_{s}}$ on $W^{\mu}$. The objects $v_{\left.P_{[}\right]}^{J}$ denote generalized Steinberg representations with respect to certain standard parabolic subgroups $P_{I_{[w]}}$ of $J$. On the other hand, the Galois group $\Gamma_{E_{s}}$ operates via Galois representations ind $[w]$. We refer to the next section for a detailed explanation of this formula.

We now mention previous results on the cohomology of period domains. The bulk of this work has been done for Drinfeld's upper half plane $\Omega^{d+1}$. The de Rham and the étale cohomology with torsion coefficients prime to $p$ of $\Omega^{d+1}$ were computed by Schneider and Stuhler in [SS]. The case of the continuous $\ell$-adic cohomology with compact support has been recently solved by Dat [D]. He makes heavy use of the specialization map of $\Omega^{d+1}$ into the Bruhat-Tits building of $\mathrm{GL}_{d+1}$. For further results on the cohomology of $\Omega^{d+1}$ dealing with $p$-adic aspects, we refer to the work of Alon and de Shalit [AS1], [AS2], Große-Klönne [G], Iovita and Spiess [IS], Ito [I], Schneider [S], de Shalit [dS1], [dS2].

For general period domains where the underlying isocrystal is basic, there exists a formula for the continuous $\ell$-adic Euler-Poincaré characteristic in the Grothendieck group of smooth $J\left(\mathbb{Q}_{p}\right) \times \operatorname{Gal}\left(\bar{E}_{s} / E_{s}\right)$-representations due to Kottwitz and Rapoport [R1], [R2]. Their approach is through the Harder-Narasimhan stratification on $\mathscr{F}^{\text {rig }}$ in which the period domain appears as an open stratum. The individual cohomology groups with torsion coefficients prime to $p$ were computed in [O2].

The present paper structures as follows. In the first section we fix notation and formulate the main result. The second section deals with the construction of the acyclic resolution of the above mentioned object in the derived category of étale sheaves on $Y^{\text {ad }}$. In the final section we compute the cohomology by evaluating the spectral sequence associated to this complex.

I wish to thank M. Rapoport for his useful comments on this paper. Furthermore, I would like to thank J.-F. Dat and A. Huber for helpful and interesting discussions.

\section{Notation and the main result}

The notation will be the same as in [O2]. So, $L$ is an algebraically closed field of characteristic $p>0, K_{0}=W(L)_{\mathbb{Q}}$ denotes the fraction field of the ring of Witt vectors and $\sigma \in \operatorname{Aut}\left(K_{0} / \mathbb{Q}_{p}\right)$ is the Frobenius automorphism. Furthermore, $\ell \in \mathbb{N}$ is a prime number with $(\ell, p)=1$. 
Let $G$ be a connected quasi-split reductive group over $\mathbb{Q}_{p}$. We fix an element $b \in G\left(K_{0}\right)$ which defines an isocrystal with $G$-structure on $L$, i.e, an exact faithful tensor functor

$$
N_{b}: \operatorname{Rep}_{\mathbb{Q}_{p}}(G) \rightarrow \operatorname{Isoc}(L)
$$

from the category of finite-dimensional rational $G$-representations into the category of isocrystals over $L$. We denote by

$$
v:=v_{b}: \mathbb{D} \rightarrow G_{K_{0}}
$$

the slope homomorphism of $N_{b}$, where $\mathbb{D}$ is the algebraic pro-torus over $\mathbb{Q}_{p}$ with character group $\mathbb{Q}$. For a representation $\rho: G \rightarrow \operatorname{GL}(V)$, the composition $\rho \circ v: \mathbb{D} \rightarrow$ $\operatorname{GL}\left(V_{K_{0}}\right)$ describes the decomposition of $N_{b}(V)$ into simple subisocrystals together with their slopes ([RR], Example 1.10). We suppose that $b$ is decent and basic. By definition ([RZ], 1.8), a decent element $b \in G\left(K_{0}\right)$ satisfies for some integer $s \in \mathbb{N}$ an equation

$$
(b \sigma)^{s}=s v_{b}(p) \sigma^{s}
$$

in the semi-direct product $G\left(K_{0}\right) \rtimes\langle\sigma\rangle$ such that $s \cdot v_{b}: \mathbb{D} \rightarrow G_{K_{0}}$ factors through $\mathbb{G}_{m}$. It follows that $b \in G\left(\mathbb{Q}_{p} s\right)$ and that $v$ is defined over $\mathbb{Q}_{p^{s}}$. The element $b$ is basic if $v$ factors through the center of $G_{K_{0}}$. An alternative definition is as follows. Let $J$ be the automorphism group of $N_{b}$, which is a reductive algebraic group over $\mathbb{Q}_{p}$. In general it is an inner form of some Levi subgroup of $G$. It is an inner form of $\mathrm{G}$ if and only if $b$ is basic.

We fix a conjugacy class

$$
\{\mu\} \subset X_{*}(G)
$$

of one-parameter subgroups (1-PS) of $G$ over the algebraic closure $\overline{\mathbb{Q}}_{p}$ of $\mathbb{Q}_{p}$. Let $E$ be the Shimura field of $\{\mu\}$. This finite extension of $\mathbb{Q}_{p}$ is the smallest extension of $\mathbb{Q}_{p}$ such that its absolute Galois group stabilizes $\{\mu\}$ as an element in the set of conjugacy classes of 1-PS of $G$. By Kottwitz's Lemma ([K2], Lemma 1.1.3), there exists a 1-PS $\mu \in\{\mu\}$ which is defined over $E$. Hence, the conjugacy class $\{\mu\}$ defines a flag variety

$$
\mathscr{F}:=\mathscr{F}(G,\{\mu\}):=G_{E} / P(\mu)
$$

defined over $E$. Here, we write $P(\lambda)$ for the parabolic subgroup of $G$ which is attached to a 1-PS $\lambda$. Let $\mathscr{F}^{\text {rig }}$ be the rigid analytic flag variety associated to $\mathscr{F}$. Put

$$
E_{s}:=E \cdot \mathbb{Q}_{p^{s}} \quad \text { and } \quad \Gamma_{E_{s}}:=\operatorname{Gal}\left(\bar{E}_{s} / E_{S}\right) .
$$

The period domain $\mathcal{F}_{b}^{\text {wa }}$ associated to the triple $(G, b,\{\mu\})$ is the set of filtrations in $\mathscr{F}^{\text {rig }}$ which are weakly admissible with respect to $N_{b}$. It has a natural structure of an admissible open rigid-analytic subset of $\left(\mathscr{F} \otimes_{E} E_{S}\right)^{\text {rig }}$ ([RZ], Proposition 1.36). Furthermore, it is a $J\left(\mathbb{Q}_{p}\right)$-invariant subset of $\mathscr{F}^{\text {rig }}$ with respect to the natural action of $J\left(\mathbb{Q}_{p}\right)$ on $\mathscr{F}^{\text {rig }}$. 
We fix a maximal $\mathbb{Q}_{p}$-split torus $S$ of the derived group $J_{\text {der }}$ of $J$. Further, we let $P_{0}$ be a minimal $\mathbb{Q}_{p}$-parabolic subgroup of $J$ such that $S \subset P_{0}$. We denote by

$$
\Delta=\left\{\alpha_{1}, \ldots, \alpha_{d}\right\} \subset X^{*}(S)
$$

the corresponding set of relative simple roots. Let

$$
\left\{\omega_{\alpha} ; \alpha \in \Delta\right\} \subset X_{*}(S)_{\mathbb{Q}}
$$

be the dual basis of $\Delta$ with respect to the natural pairing $X_{*}(S) \times X^{*}(S) \rightarrow \mathbb{Z}$. Let $T$ be a maximal torus of $G$ which contains $S$ and such that $\mu, v \in X_{*}(T)_{\mathbb{Q}} \cong$ $\operatorname{Hom}_{K_{0}}(\mathbb{D}, T)$. We fix a Borel subgroup $B$ of $G$ such that

- $B \subset P\left(\omega_{\alpha}\right)$ for all $\alpha \in \Delta$,

- $\mu$ lies in the positive Weyl chamber with respect to $B$.

We may assume that $\Delta$ is given by restriction of a root basis of $G$ with respect to $B \supset T$. Put

$$
\bar{\mu}:=\frac{1}{\left|\operatorname{Gal}\left(E / \mathbb{Q}_{p}\right)\right|} \sum_{\gamma \in \operatorname{Gal}\left(E / \mathbb{Q}_{p}\right)} \gamma \mu .
$$

By $[\mathrm{FR}]$ we know that $\widetilde{F}_{b}^{\text {wa }}$ is non-empty if and only if

$$
\bar{\mu} \geq v
$$

with respect to the dominance order $\geq$ on $X_{*}(T)_{\mathbb{Q}}$ induced by $B$. In our situation of a basic isocrystal, the latter condition simply means that $\bar{\mu}-v \in X_{*}\left(T_{\mathrm{der}}\right)$, where $T_{\text {der }} \subset T$ is the maximal torus of the derived group $G_{\text {der }}$ of $G$.

Let $W$ the Weyl group of $G$. Denote by $W_{\mu} \subset W$ the stabilizer of $\mu$, and by $W^{\mu}$ the set of Kostant representatives with respect to $W / W_{\mu}$. We have an action of $\Gamma_{E_{s}}$ on $W$ which preserves $W^{\mu}$. For any orbit $[w] \in W^{\mu} / \Gamma_{E_{s}}$, we have the induced representation

$$
\operatorname{ind}_{[w]}:=\operatorname{Ind}_{\operatorname{Stab}_{\Gamma_{E_{S}}}(w)}^{\Gamma_{E_{S}}}\left(\mathbb{Q}_{\ell}\right)
$$

of $\Gamma_{E_{s}}$, where we consider the trivial action of $\operatorname{Stab}_{\Gamma_{E_{S}}}(w)$ on $\mathbb{Q}_{\ell}$. For any subset $I \subset \Delta$, we set

$$
\Omega_{I}:=\left\{[w] \in W^{\mu} / \Gamma_{E_{s}} ;\left(w \mu, \omega_{\alpha}\right)>\left(\nu, \omega_{\alpha}\right) \text { for all } \alpha \notin I\right\} .
$$

Here, we have fixed an invariant inner product (, ) on $G$ (see [T], Section 7). Finally, we denote for $[w] \in W^{\mu} / \Gamma_{E_{s}}$, by $I_{[w]}$ the minimal subset of $\Delta$ such that $[w]$ is contained in $\Omega_{I_{[w]}}$.

For a parabolic subgroup $P \subset J$ defined over $\mathbb{Q}_{p}$, let

$$
i_{P}^{J}=C^{\infty}\left(J\left(\mathbb{Q}_{p}\right) / P\left(\mathbb{Q}_{p}\right), \mathbb{Q}_{\ell}\right)
$$


be the smooth representation of $J\left(\mathbb{Q}_{p}\right)$ consisting of locally constant $\mathbb{Q}_{\ell}$-valued functions on the $p$-adic manifold $J\left(\mathbb{Q}_{p}\right) / P\left(\mathbb{Q}_{p}\right)$. The generalized Steinberg representation $v_{P}^{J}$ is defined by

$$
v_{P}^{J}=i_{P}^{J} / \sum_{P \subsetneq Q} i_{Q}^{J} .
$$

This is an irreducible smooth admissible representation of $J\left(\mathbb{Q}_{p}\right)$ ([C], Theorem 1.1). Finally, for $I \subset \Delta$ we put

$$
P_{I}:=\bigcap_{\alpha \notin I} P^{J}\left(\omega_{\alpha}\right)
$$

which is a standard-parabolic subgroup of $J$ defined over $\mathbb{Q}_{p}$. The extreme cases are $P_{\Delta}=J$ and $P_{\emptyset}=P_{0}$. Here, $P^{J}(\lambda)$ is the parabolic subgroup of $J$ attached to a 1-PS $\lambda$ of $J$.

We denote by $H_{c}^{*}\left(\mathcal{F}_{b}^{\text {wa }}, \mathbb{Q}_{\ell}\right)$ the continuous $\ell$-adic cohomology with compact support of $\mathscr{F}_{b}^{\text {wa }}$. We refer to Section 3 for its definition. We have a natural action of $J\left(\mathbb{Q}_{p}\right) \times \Gamma_{E_{s}}$ on $H_{c}^{*}\left(\mathcal{F}_{b}^{\text {wa }}, \mathbb{Q}_{\ell}\right)$ which is smooth in the sense of $p$-adic Lie group representations. In fact, in the case of finite cohomology coefficients this statement is due to Berkovich [B1]. In our situation, there are unpublished notes [B2] of Berkovich treating our case. In any case, we first follow [B1], Proposition 6.4 to deduce that the action of $J\left(\mathbb{Q}_{p}\right) \times \Gamma_{E_{s}}$ on $\mathcal{F}_{b}^{\text {wa }}$ is continuous. Then we apply Fargue's result [F], Corollary 4.1.19 to conclude that $H_{c}^{*}\left(\mathcal{F}_{b}^{\text {wa }}, \mathbb{Q}_{\ell}\right)$ is a smooth representation.

Our result is the following theorem.

Theorem 1.1. Let $b \in G\left(K_{0}\right)$ be a basic element such that $\bar{\mu}-v \in X_{*}\left(T_{\mathrm{der}}\right)$. We have for the compactly supported continuous $\ell$-adic cohomology of $\mathcal{F}_{b}^{\text {wa }}$ the following formula as representations of $J\left(\mathbb{Q}_{p}\right) \times \Gamma_{E_{s}}$ :

$$
H_{c}^{*}\left(\mathcal{F}_{b}^{\text {wa }}, \mathbb{Q}_{\ell}\right)=\bigoplus_{[w] \in W^{\mu} / \Gamma_{E_{s}}} v_{P_{[w]}}^{J} \otimes \operatorname{ind}_{[w]}\{-l([w])\}\left[-\left|\Delta \backslash I_{[w]}\right|\right] .
$$

Here for a representation $V$, we have set $V\{-m\}=V(-m)[-2 m], m \in \mathbb{Z}$. The symbol $(m)$ denotes the $m$-th Tate twist and $[-m]$ symbolizes that the corresponding module is shifted into degree $m$ of the graded cohomology ring.

\section{The fundamental complex}

In [O2] we constructed an acyclic complex of étale sheaves on the complement of $\mathcal{F}_{b}^{\text {wa }}$ in $\mathscr{F}$. Strictly speaking, we formed the complement in the category of Huber's adic spaces [H1], i.e., we set

$$
Y^{\mathrm{ad}}:=\mathscr{F}^{\mathrm{ad}} \backslash\left(\mathcal{F}_{b}^{\mathrm{wa}}\right)^{\mathrm{ad}} .
$$


Here, 'ad' indicates the adic space attached to a scheme or a rigid-analytic variety. The advantage of this larger category is that $Y^{\text {ad }}$ has the structure of a closed pseudo-adic subspace of $\mathcal{F}^{\text {ad }}$ for which there exists an étale site and hence a topos as well (compare

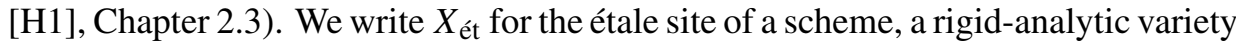
or that of a pseudo-adic space $X$.

In [H2] (see Chapter 1) Huber introduced the compactly supported cohomology of $\ell$-adic sheaves on adic spaces. We briefly explain Huber's definition. Let $R$ be a complete discrete valuation ring with maximal ideal $\mathfrak{m}$ and with $\operatorname{char}(R / \mathfrak{m})>0$. Let $X$ be a taut separated pseudo-adic space locally of ${ }^{+}$weakly finite type over an analytic algebraically closed affinoid field $\mathrm{Spa}\left(k, k^{0}\right)$. A $R_{\bullet}$-module on $X_{\text {ét }}$ is a projective system

$$
\cdots \rightarrow F_{n+1} \rightarrow F_{n} \rightarrow \cdots \rightarrow F_{1}
$$

of $R$-modules on $X_{\text {ét }}$ such that $\mathfrak{m}^{n} \cdot F_{n}=0$ for every $n \in \mathbb{N}$. Denote by $\bmod \left(X_{\text {ét }}-R_{\bullet}\right)$ the category of $R_{\bullet}$-modules on $X_{\text {ét. }}$. This is an abelian category with enough injectives. Further, we let $\bmod (R) \operatorname{resp} . \bmod \left(X_{\text {ét }}-R\right)$ be the category of $R$-modules resp. $R$-modules on $X_{\text {ét }}$. For any $R_{\bullet}$-module $\left(F_{n}\right)_{n}$ on $X_{\text {ét }}$, the compactly supported cohomology $H_{c}^{p}\left(X,\left(F_{n}\right)_{n}\right)$ is defined as follows. In the case where $X$ is partially complete, the cohomology is given by

$$
H_{c}^{p}\left(X,\left(F_{n}\right)_{n}\right):=R^{p} \Gamma_{c}\left(X,\left(F_{n}\right)_{n}\right), \quad p \in \mathbb{N} .
$$

Here $\Gamma_{c}$ is the left exact functor

$$
\begin{aligned}
\Gamma_{c}: \bmod \left(X_{\text {ét }}-R_{\bullet}\right) & \rightarrow \bmod (R), \\
\left(F_{n}\right)_{n} & \mapsto \Gamma_{c}\left(X, \lim _{\longleftarrow} F_{n}\right),
\end{aligned}
$$

where $\Gamma_{c}\left(X, \lim F_{n}\right)$ is the $R$-module of global sections whose support is complete over $\operatorname{Spa}\left(k, k^{0}\right)$. In general, if $X$ is not partially complete, one defines the compactly supported cohomology using a partially complete compactification of $X$. Finally, by the continuous $\ell$-adic cohomology with compact support of $X$ we mean the $\mathbb{Q}_{\ell}$-vector spaces

$$
H_{c}^{*}\left(X, \mathbb{Q}_{\ell}\right)=H_{c}^{*}\left(X,\left(\mathbb{Z} / \ell^{n} \mathbb{Z}\right)_{n}\right) \otimes_{\mathbb{Z}_{\ell}} \mathbb{Q}_{\ell} .
$$

In the case of algebraic varieties this definition is due to Jannsen [J]. Huber does not use this notation in [H2].

We indicate briefly the relation to Berkovich's definition of compactly supported cohomology of $R_{\bullet}$-modules which applies to his analytic spaces. Berkovich defines this cohomology by using a different global section functor. It is given by

$$
\begin{aligned}
& \bmod \left(X_{\text {ét }}-R_{\bullet}\right) \rightarrow \bmod (R), \\
& \left(F_{n}\right)_{n} \mapsto \underset{U \in \mathbb{U}}{\lim _{n \in \mathbb{N}}} \lim _{c} \Gamma_{c}\left(U, F_{n}\right)
\end{aligned}
$$


Here $\mathbb{U}$ is the set of distinguished open subsets in the analytic space $X$. In his paper ([F], 4.1.2), Fargues uses Huber's definition of compactly supported cohomology of $R_{\bullet}$-modules in the case of analytic spaces. He proves that it coincides with that of Berkovich. For rigid-analytic varieties, this statement was already shown in [H2], Proposition 1.5.

Consider the left exact functors

$$
\begin{aligned}
\pi_{*}: \bmod \left(X_{\text {ét }}-R_{\bullet}\right) & \rightarrow \bmod \left(X_{\text {ét }}-R\right), \\
\left(F_{n}\right)_{n} & \mapsto \lim _{n} F_{n}
\end{aligned}
$$

and

$$
\begin{aligned}
\Gamma_{!}: \bmod \left(X_{\text {ét }}-R\right) & \rightarrow \bmod (R), \\
F & \mapsto \Gamma_{c}(F) .
\end{aligned}
$$

If $X$ is partially complete, then, by definition, we have $\Gamma_{c}=\Gamma_{!} \circ \pi_{*}$. This identity then gives rise to an identity (compare [H2], Lemma 2.3 (i))

$$
R^{+} \Gamma_{c}=R^{+} \Gamma_{!} \circ R^{+} \pi_{*}
$$

of exact functors from the derived category $D^{\geq 0}\left(\bmod \left(X_{\text {ét }}-R_{\bullet}\right)\right)$ into $D^{\geq 0}(\bmod (R))$.

For any rational 1-PS $\lambda \in X_{*}(J)_{\mathbb{Q}}$ of $J$, we consider in [O2] the closed $E_{s}$-subvariety $Y_{\lambda}$ of $\mathcal{F}$ consisting of points where $\lambda$ violates the semi-stability condition. By [O2], Corollary 2.4 , we have the identity

$$
Y^{\mathrm{ad}}=\bigcup_{\lambda} Y_{\lambda}^{\mathrm{ad}}
$$

of pseudo-adic spaces. Furthermore, we define for any subset $I \subset \Delta$ the closed subvariety

$$
Y_{I}:=\bigcap_{\alpha \notin I} Y_{\omega_{\alpha}}
$$

of $\mathcal{F}$, on which we have an action of the $p$-adic group $P_{I}\left(\mathbb{Q}_{p}\right)$. For any compact open subset $W \subset J / P_{I}\left(\mathbb{Q}_{p}\right)$, we put

$$
Z_{I}^{W}:=\bigcup_{g \in W} g Y_{I}^{\mathrm{ad}}
$$

Then by [O2], Lemma 3.2, we know that $Z_{I}^{W}$ is a closed pseudo-adic subspace of $\mathscr{F}^{\text {ad }}$. By (2) it follows that

$$
Y^{\mathrm{ad}}=\bigcup_{\substack{I \subset \Delta \\|\Delta \backslash I|=1}} Z_{I}^{J / P_{I}\left(\mathbb{Q}_{p}\right)}
$$


Starting from a subset $I \subset \Delta$ and the constant étale sheaf $\mathbb{Z} / n \mathbb{Z}, n \in \mathbb{N}$, on $Y^{\text {ad }}$ we constructed in loc. cit. a sheaf of locally constant sections on the same space. Actually, the same construction goes through if we start with an arbitrary étale sheaf $F$ on $Y^{\text {ad }}$. We recall the definition. Let

$$
\Phi_{g, I}: g Y_{I}^{\mathrm{ad}} \rightarrow Y^{\mathrm{ad}}
$$

resp.

$$
\tilde{\Phi}_{g, I, W}: g Y_{I}^{\mathrm{ad}} \rightarrow Z_{I}^{W}
$$

resp.

$$
\Psi_{I, W}: Z_{I}^{W} \rightarrow Y^{\text {ad }}
$$

the natural closed embeddings of pseudo-adic spaces. Let $F$ be a $R$-module on $Y_{\mathrm{et}}^{\mathrm{ad}}$. Put

$$
F_{g, I}:=\left(\Phi_{g, I}\right)_{*}\left(\Phi_{g, I}^{*} F\right)
$$

resp.

$$
F_{Z_{I}^{W}}:=\left(\Psi_{I, W}\right)_{*}\left(\Psi_{I, W}^{*} F\right)
$$

and let

$$
\tilde{\Phi}_{g, I, W}^{\#}: F_{Z_{I}^{W}} \rightarrow F_{g, I}
$$

be the natural homomorphism given by restriction. We denote by

$$
\prod_{g \in J / P_{I}\left(\mathbb{Q}_{p}\right)}^{\prime} F_{g, I}
$$

the $R$-submodule of $\prod_{g \in J / P_{I}\left(\mathbb{Q}_{p}\right)} F_{g, I}$, which is defined as the sheaf associated to the following presheaf ${ }^{P} \prod_{g \in J / P_{I}\left(\mathbb{Q}_{p}\right)}^{\prime} F_{g, I}$. For any element $U \rightarrow Y^{\text {ad }}$ of the étale site $Y_{\text {ét }}^{\text {ad }}$, we put

$$
\begin{aligned}
\left(\prod_{g \in J / P_{I}\left(\mathbb{Q}_{p}\right)}^{P} F_{g, I}\right)(U):= & \left(s_{g}\right)_{g} \in \prod_{g \in J / P_{I}\left(\mathbb{Q}_{p}\right)} F_{g, I}(U) ; \text { there exists a (finite) } \\
& \text { disjoint covering } J / P_{I}\left(\mathbb{Q}_{p}\right)=\bigcup_{j \in A} W_{j} W_{j} \text { by compact } \\
& \text { open subsets and sections } s_{j} \in F_{Z_{I}}(U), j \in A, \text { such } \\
& \text { that } \left.\tilde{\Phi}_{g, I, W_{j}}^{\#}\left(s_{j}\right)=s_{g} \text { for all } g \in W_{j}\right\} .
\end{aligned}
$$

We call $\prod_{g \in J / P_{I}\left(\mathbb{Q}_{p}\right)}^{\prime} F_{g, I}$ the subsheaf of locally constant sections of $\prod_{g \in J / P_{I}\left(\mathbb{Q}_{p}\right)} F_{g, I}$.

Remark. If we work with the restricted étale site $Y_{\text {et, f.p. }}^{\text {ad }}$ consisting of objects $U$ in $Y_{\text {ét }}^{\text {ad }}$ where the structure morphism $U \rightarrow Y^{\text {ad }}$ is quasi-compact and quasi-separated, it is easy to see that the presheaf ${ }^{P} \prod_{g \in J / P_{I}\left(\mathbb{Q}_{p}\right)}^{\prime} F_{g, I}$ is already a sheaf (compare also [O2]). 
The sheaf $\prod_{g \in J / P_{I}\left(\mathbb{Q}_{p}\right)}^{\prime} F_{g, I}$ may be viewed as an inductive limit of sheaves. In fact, let $\mathcal{C}_{I}$ be the category of compact open disjoint coverings of $J / P_{I}\left(\mathbb{Q}_{p}\right)$ in which the morphisms are given by the refinement-order. For a covering $c=\left(W_{j}\right)_{j \in A} \in \mathcal{C}_{I}$, we denote by $F_{c}$ the sheaf defined by

$$
\begin{array}{r}
F_{c}(U):=\left\{\left(s_{g}\right)_{g} \in \prod_{g \in J / P_{I}\left(\mathbb{Q}_{p}\right)} F_{g, I}(U) ; \text { there are sections } s_{j} \in F_{Z_{I}^{W_{j}}}(U), j \in A,\right. \\
\text { such that } \left.\tilde{\Phi}_{g, I, W_{j}}^{\#}\left(s_{j}\right)=s_{g} \text { for all } g \in W_{j}\right\} .
\end{array}
$$

Alternatively, one can define $F_{c}$ as the image of the natural morphism of sheaves

$$
\bigoplus_{j \in A} F_{Z_{I}^{W_{j}} \hookrightarrow} \prod_{g \in J / P_{I}\left(\mathbb{Q}_{p}\right)} F_{g, I}
$$

Then we may write

$$
\prod_{g \in J / P_{I}\left(\mathbb{Q}_{p}\right)}^{\prime} F_{g, I}=\lim _{c \in \mathbb{C}_{I}} F_{c}
$$

Our construction extends naturally to the derived category of étale sheaves on $Y^{\text {ad }}$. Indeed, the assignment $F \mapsto \prod_{g \in J / P_{I}\left(\mathbb{Q}_{p}\right)}^{\prime} F_{g, I}$ is functorial and induces therefore a functor

$$
\prod_{g \in J / P_{I}\left(\mathbb{Q}_{p}\right)}^{\prime}: \bmod \left(Y_{\text {ét }}^{\mathrm{ad}}-R\right) \rightarrow \bmod \left(Y_{\text {ét }}^{\mathrm{ad}}-R\right),
$$

which is easily seen to be exact. Thus, we obtain an exact functor on the corresponding derived categories

$$
\prod_{g \in J / P_{I}\left(\mathbb{Q}_{p}\right)}^{\prime}=R^{+} \prod_{g \in J / P_{I}\left(\mathbb{Q}_{p}\right)}^{\prime}: D^{\geq 0}\left(\bmod \left(Y_{\text {ét }}^{\mathrm{ad}}-R\right)\right) \rightarrow D^{\geq 0}\left(\bmod \left(Y_{\text {ét }}^{\mathrm{ad}}-R\right)\right) .
$$

From now on we let $R=\mathbb{Z}_{\ell}$. Furthermore, we consider the $\mathbb{Z}_{\ell \bullet}$-module

$$
\left(F_{n}\right)_{n}=\left(\mathbb{Z} / \ell^{n} \mathbb{Z}\right)_{n} \in \bmod \left(\mathcal{F}^{\mathrm{ad}}-\mathbb{Z}_{\ell}\right) .
$$

Let $i: Y^{\text {ad }} \hookrightarrow \mathscr{F}^{\text {ad }}$ be the inclusion. Put

$$
\Upsilon:=i^{*} R^{+} \pi_{*}\left(\mathbb{Z} / \ell^{n} \mathbb{Z}\right)_{n} \in D^{\geq 0}\left(\bmod \left(Y_{\text {ét }}^{\text {ad }}-\mathbb{Z}_{\ell}\right)\right) .
$$

By [H2], Proposition 2.6 (ii), we have a long exact sequence

$$
\begin{aligned}
\cdots & \rightarrow H_{c}^{p}\left(\left(\mathcal{F}^{\mathrm{wa}}\right)^{\mathrm{ad}},\left(\mathbb{Z} / \ell^{n} \mathbb{Z}\right)_{n}\right) \rightarrow H_{c}^{p}\left(\mathcal{F}^{\mathrm{ad}},\left(\mathbb{Z} / \ell^{n} \mathbb{Z}\right)_{n}\right) \rightarrow H_{c}^{p}\left(Y^{\mathrm{ad}}, \Upsilon\right) \\
& \rightarrow H_{c}^{p+1}\left(\left(\mathcal{F}^{\mathrm{wa}}\right)^{\mathrm{ad}},\left(\mathbb{Z} / \ell^{n} \mathbb{Z}\right)_{n}\right) \rightarrow \cdots
\end{aligned}
$$


Vol. 82 (2007)

Taking Huber's comparison theorem for schemes into account ([H2], Theorem 4.2) we have

$$
H_{c}^{*}\left(\mathscr{F}^{\mathrm{ad}},\left(\mathbb{Z} / \ell^{n} \mathbb{Z}\right)_{n}\right)=H_{c}^{*}\left(\mathscr{F},\left(\mathbb{Z} / \ell^{n} \mathbb{Z}\right)_{n}\right) .
$$

On the other hand, by Jannsen's comparison theorem ([J], Remark 3.5) we conclude that

$$
H_{c}^{*}\left(\mathcal{F},\left(\mathbb{Z} / \ell^{n} \mathbb{Z}\right)_{n}\right)=H_{c}^{*}\left(\mathcal{F}, \mathbb{Z}_{\ell}\right)=H^{*}\left(\mathcal{F}, \mathbb{Z}_{\ell}\right),
$$

where $H^{*}\left(\mathcal{F}, \mathbb{Z}_{\ell}\right):=\lim _{n} H^{*}\left(\mathcal{F}, \mathbb{Z} / \ell^{n} \mathbb{Z}\right)$ denotes the $\ell$-adic cohomology of the scheme $\mathscr{F}$. Further, by the very definition of the compactly supported $\ell$-adic cohomology of rigid-analytic varieties we have

$$
H_{c}^{*}\left(\left(\mathcal{F}^{\mathrm{wa}}\right)^{\mathrm{ad}},\left(\mathbb{Z} / \ell^{n} \mathbb{Z}\right)_{n}\right)=H_{c}^{*}\left(\mathcal{F}^{\mathrm{wa}},\left(\mathbb{Z} / \ell^{n} \mathbb{Z}\right)_{n}\right) .
$$

Therefore, in order to prove Theorem 1.1 it suffices to compute the cohomology groups

$$
H_{c}^{p}\left(Y^{\mathrm{ad}}, \Upsilon\right)=H^{p}\left(Y^{\mathrm{ad}}, \Upsilon\right)
$$

Let $F$ be a étale sheaf on $Y^{\text {ad }}$. In [O2] we constructed in the case $F=\mathbb{Z} / n \mathbb{Z}$ a complex of étale sheaves on $Y^{\text {ad }}$ :

$$
\begin{aligned}
0 \rightarrow F \rightarrow & \bigoplus_{\substack{I \subset \Delta \\
|\Delta \backslash I|=1}} \prod_{g \in J / P_{I}\left(\mathbb{Q}_{p}\right)}^{\prime} F_{g, I} \rightarrow \bigoplus_{\substack{I \subset \Delta \\
|\Delta \backslash I|=2}} \prod_{g \in J / P_{I}\left(\mathbb{Q}_{p}\right)}^{\prime} F_{g, I} \rightarrow \cdots \\
& \cdots \rightarrow \bigoplus_{\substack{I \subset \Delta \\
|\Delta \backslash I|=d-1}} \prod_{g \in J / P_{I}\left(\mathbb{Q}_{p}\right)}^{\prime} F_{g, I} \rightarrow \prod_{g \in J / P_{\emptyset}\left(\mathbb{Q}_{p}\right)}^{\prime} F_{g, \emptyset} \rightarrow 0
\end{aligned}
$$

The same construction works for arbitrary étale sheaves $F$ on $Y^{\text {ad }}$ as well. Furthermore, we proved (loc. cit. Theorem 3.3) that the complex above is acyclic in the case $F=\mathbb{Z} / n \mathbb{Z}$. The same holds if we consider overconvergent étale sheaves $F$ on $Y^{\text {ad }}$. Recall that an étale sheaf $F$ on a pseudo-adic space $X$ is called overconvergent if the specialization morphisms $F_{\xi_{1}} \rightarrow F_{\xi_{2}}$ are isomorphisms for all pairs of geometric points $\xi_{1}, \xi_{2}$ of $X$ such that $\xi_{1}$ is a specialization of $\xi_{2}$.

Theorem 2.1. Let $F$ be an overconvergent étale sheaf on $Y^{\text {ad }}$. Then the complex $(*)$ is acyclic.

Proof. The proof is almost the same as that of Theorem 3.3 in [O2]. First, we follow the proof of Lemma 3.4 in loc. cit. to show that the complex $(*)$ consists of overconvergent sheaves. Further, by localizing in a maximal geometric point $\xi$ of $Y^{\text {ad }}$ we obtain a chain complex with values in $F_{\xi}$ instead of $\mathbb{Z} / n \mathbb{Z}$. In loc. cit. we reduced the acyclicity of this chain complex in the case $F=\mathbb{Z} / n \mathbb{Z}$ to the contractibility of its index set. The same argument applies in our general case. 
Choose a complex $\bar{\Upsilon}$ representing the object $\Upsilon \in D^{\geq 0}\left(\bmod \left(Y_{\text {ét }}^{\text {ad }}-\mathbb{Z}_{\ell}\right)\right.$. Substituting the étale sheaf $F$ in $(*)$ by $\bar{\Upsilon}$ we obtain a complex in the category of complexes with values in $\bmod \left(Y_{\text {ét }}^{\text {ad }}-\mathbb{Z}_{\ell}\right)$ :

$$
\begin{aligned}
0 \rightarrow \bar{\Upsilon} & \rightarrow \bigoplus_{\substack{I \subset \Delta \\
|\Delta \backslash I|=1}} \prod_{g \in J / P_{I}\left(\mathbb{Q}_{p}\right)}^{\prime} \bar{\Upsilon}_{g, I} \rightarrow \bigoplus_{\substack{I \backslash \Delta \\
|\Delta \backslash I|=2}} \prod_{g \in J / P_{I}\left(\mathbb{Q}_{p}\right)}^{\prime} \bar{\Upsilon}_{g, I} \rightarrow \ldots \\
& \cdots \rightarrow \bigoplus_{\substack{I \subset \Delta \\
|\Delta \backslash I|=d-1}} \prod_{g \in J / P_{I}\left(\mathbb{Q}_{p}\right)}^{\prime} \bar{\Upsilon}_{g, I} \rightarrow \prod_{g \in J / P_{\emptyset}\left(\mathbb{Q}_{p}\right)}^{\prime} \bar{\Upsilon}_{g, \emptyset} \rightarrow 0 .
\end{aligned}
$$

Corollary 2.2. The complex (**) is exact.

Proof. By Theorem 2.1 we merely have to assure that the individual contributions of $\Upsilon=i^{*} R^{+} \pi_{*}\left(\mathbb{Z} / \ell^{n} \mathbb{Z}\right)_{n}$ are overconvergent. Since $Y^{\text {ad }}$ is a closed subspace of $\mathcal{F}^{\text {ad }}$, it is enough to prove the assertion for the complex $R^{+} \pi_{*}\left(\mathbb{Z} / \ell^{n} \mathbb{Z}\right)_{n}$ on $\mathcal{F}^{\text {ad }}$. The étale topoi of $\mathscr{F}^{\text {ad }}$ and $\mathcal{F}^{\text {rig }}$ are equivalent [H1] Proposition 2.1.4. Let $\mathscr{F}^{\text {an }}$ be Berkovich's analytic space attached to $\mathscr{F}$. There is an equivalence between the étale topos of $\mathscr{F}^{\text {an }}$ and the étale subtopos of $\mathscr{F}^{\text {rig }}$ consisting of overconvergent sheaves (loc. cit. Proposition 8.3.3). The claim follows now easily by comparison.

\section{The computation}

In this final section we evaluate the spectral sequence which is induced by the complex $(* *)$. This computation ends with the determination of the hypercohomology of $\Upsilon$. The proof of Theorem 1.1 then follows from the long exact cohomology sequence (5). The arguments of this final section are modelled on the results in [O2], Section 4 , treating the torsion sheaf $\mathbb{Z} / n \mathbb{Z}$ instead of $\Upsilon$.

For the next two statements we fix a subset $I \subsetneq \Delta$.

Proposition 3.1. We have

$$
H_{c}^{*}\left(Y_{I}^{\mathrm{ad}}, \mathbb{Q}_{\ell}\right)=\bigoplus_{[w] \in \Omega_{I}} \operatorname{ind}_{[w]}\{-l[w]\}
$$

Proof. By the comparison theorems of Huber (see [H2], Theorem 4.2) resp. Jannsen (see [J], Remark 3.5), we have $H_{c}^{*}\left(Y_{I}^{\text {ad }},\left(\mathbb{Z} / \ell^{n} \mathbb{Z}\right)_{n}\right)=H_{c}^{*}\left(Y_{I},\left(\mathbb{Z} / \ell^{n} \mathbb{Z}\right)_{n}\right)$ resp. $H_{c}^{*}\left(Y_{I},\left(\mathbb{Z} / \ell^{n} \mathbb{Z}\right)_{n}\right)=H^{*}\left(Y_{I}, \mathbb{Z}_{\ell}\right)$. Then we proceed as in Proposition 4.2 [O2].

Next we compute the cohomology of the ingredients in $(* *)$, i.e., the hypercohomology of the objects $\prod_{g \in J / P_{I}\left(\mathbb{Q}_{p}\right)}^{\prime} \Upsilon_{g, I}$. 
Vol. 82 (2007)

Proposition 3.2. We have

$$
H^{i}\left(Y^{\mathrm{ad}}, \prod_{g \in J / P_{I}\left(\mathbb{Q}_{p}\right)}^{\prime} \Upsilon_{g, I}\right)=C^{\infty}\left(J / P_{I}\left(\mathbb{Q}_{p}\right), H^{i}\left(Y_{I}^{\mathrm{ad}}, \mathbb{Z}_{\ell}\right)\right)
$$

for all $i \in \mathbb{N}$. Here, $C^{\infty}\left(J / P_{I}\left(\mathbb{Q}_{p}\right), H^{i}\left(Y_{I}^{\mathrm{ad}}, \mathbb{Z}_{\ell}\right)\right)$ denotes the space of locally constant functions on $J / P_{I}\left(\mathbb{Q}_{p}\right)$ with values in $H^{i}\left(Y_{I}^{\mathrm{ad}}, \mathbb{Z}_{\ell}\right)$.

Proof. The proof is similar to Proposition 4.3 in loc. cit. Again, we fix as in the construction of (**) a representative $\bar{\Upsilon}$ of $\Upsilon$. First of all, we have by (4) the identity

$$
\prod_{g \in J / P_{I}\left(\mathbb{Q}_{p}\right)}^{\prime} \bar{\Upsilon}_{g, I}=\underset{c \in \lim _{I}}{\vec{\Upsilon}_{c}}
$$

Since $Y^{\text {ad }}$ is quasi-compact and $\mathcal{C}_{I}$ is pseudo-filtered, we get (compare also [H1], 2.3.13)

$$
H^{i}\left(Y^{\mathrm{ad}}, \prod_{g \in J / P_{I}\left(\mathbb{Q}_{p}\right)}^{\prime} \Upsilon_{g, I}\right)={\underset{c \in \mathbb{C}}{\lim _{I}}}_{H^{i}}\left(Y^{\mathrm{ad}}, \Upsilon_{c}\right)
$$

But by (2) we have a decomposition $\bar{\Upsilon}_{c} \cong \bigoplus_{W \in c} \bar{\Upsilon}_{Z_{I}^{W}}$. Thus, we obtain

$$
H^{i}\left(Y^{\mathrm{ad}}, \Upsilon_{c}\right)=\bigoplus_{W \in c} H^{i}\left(Z_{I}^{W}, \Upsilon_{\mid Z_{I}^{W}}\right)
$$

Let $\left(W_{s}\right)_{s \in \mathbb{N}}$ be a cofinal family of compact open neighbourhoods of the base point $1 \cdot P_{I}$ in the $p$-adic manifold $J / P_{I}\left(\mathbb{Q}_{p}\right)$. By Lemma 4.4 of loc. cit. we have the identity

$$
\bigcap_{s \in \mathbb{N}} Z_{I}^{W^{s}}=Y_{I}^{\mathrm{ad}}
$$

Now, we apply [H1], 2.4.6 ${ }^{1}$, to conclude that

$$
\underset{s \in \mathbb{N}}{\lim _{\vec{f}}} H^{i}\left(Z_{I}^{W^{s}}, \Upsilon_{\mid Z_{I}^{W^{s}}}\right)=H^{i}\left(Y_{I}^{\mathrm{ad}}, \Upsilon_{\mid Y_{I}^{\mathrm{ad}}}\right)
$$

Using (1) we see that

$$
H^{i}\left(Y_{I}^{\mathrm{ad}}, \Upsilon_{\mid Y_{I}^{\mathrm{ad}}}\right)=H^{i}\left(Y_{I}^{\mathrm{ad}},\left(\mathbb{Z} / \ell^{n} \mathbb{Z}\right)_{n}\right)
$$

so that by reapplying Huber's comparison theorem ([H2], Theorem 4.2) we obtain

$$
H^{i}\left(Y_{I}^{\mathrm{ad}}, \Upsilon_{\mid Y_{I}^{\mathrm{ad}}}\right)=H^{i}\left(Y_{I}^{\mathrm{ad}}, \mathbb{Z}_{\ell}\right)
$$

\footnotetext{
${ }^{1}$ Actually the statement there is only formulated for sheaves. But it is easy to see that the argument generalizes to the derived category.
} 
Combining these facts we get

$$
\begin{aligned}
H^{i}\left(Y^{\mathrm{ad}}, \prod_{g \in J / P_{I}\left(\mathbb{Q}_{p}\right)}^{\prime} \Upsilon_{g, I}\right) & =\underset{c \in \mathbb{C}_{I}}{\lim _{W \in c}} \bigoplus_{c}^{i}\left(Z_{I}^{W}, \Upsilon_{\mid Z_{I}^{W}}\right) \\
& =C^{\infty}\left(J / P_{I}\left(\mathbb{Q}_{p}\right), H^{i}\left(Y_{I}^{\mathrm{ad}}, \mathbb{Z}_{\ell}\right)\right)
\end{aligned}
$$

Consider the spectral sequence induced by the acyclic complex $(* *)$ :

$$
E_{1}^{p, q}=H_{c}^{q}\left(Y^{\mathrm{ad}}, \bigoplus_{\substack{I \subset \Delta \\|\Delta \backslash I|=p+1}} \prod_{g \in J / P_{I}\left(\mathbb{Q}_{p}\right)}^{\prime} \Upsilon_{g, I}\right) \otimes_{\mathbb{Z}_{\ell}} \mathbb{Q}_{\ell} \Longrightarrow H_{c}^{p+q}\left(Y^{\mathrm{ad}}, \Upsilon\right) \otimes_{\mathbb{Z}_{\ell}} \mathbb{Q}_{\ell}
$$

From the previous proposition we deduce that

$$
E_{1}^{p, q}=\bigoplus_{\substack{I \subset \Delta \\|\Delta \backslash I|=p+1}} C^{\infty}\left(J / P_{I}\left(\mathbb{Q}_{p}\right), H^{q}\left(Y_{I}^{\mathrm{ad}}, \mathbb{Q}_{\ell}\right)\right)
$$

As in [O2], we obtain by Proposition 3.1 a decomposition into subcomplexes

$$
E_{1}=\bigoplus_{[w] \in W^{\mu} / \Gamma_{E_{s}}} E_{1,[w]}
$$

where $E_{1,[w]}$ is the complex

$$
\left.\left(\bigoplus_{\substack{I_{[w]} \subset I \\|\Delta| I \mid=1}} i_{P_{I}}^{J} \otimes \operatorname{ind}_{[w]} \rightarrow \bigoplus_{\substack{I_{[w] \subset C} \\|\Delta \backslash I|=2}} i_{P_{I}}^{J} \otimes \operatorname{ind}_{[w]} \rightarrow \cdots \rightarrow i_{P_{[}[w]}^{J} \otimes \operatorname{ind}_{[w]}\right)\right)\{-l([w])\}
$$

By applying Proposition 4.5 of loc. cit., we obtain for the second stage of the spectral sequence $E_{2}=\bigoplus_{[w] \in W^{\mu} / \Gamma_{E_{s}}} E_{2,[w]}$ the following expression:

$$
E_{2,[w]}^{0, r}= \begin{cases}i_{P_{[w]}}^{J} \otimes \operatorname{ind}_{[w]}(-l([w])), & \left|\Delta \backslash I_{[w]}\right|=1,2 l([w])=r, \\ i_{J}^{J} \otimes \operatorname{ind}_{[w]}(-l([w])), & \left|\Delta \backslash I_{[w]}\right|>1,2 l([w])=r, \\ 0 & \text { otherwise, }\end{cases}
$$

respectively for $p>0$

$$
E_{2,[w]}^{p, r-p}= \begin{cases}v_{P_{I_{[w]}}^{J}}^{J} \otimes \operatorname{ind}_{[w]}(-l([w])), & p=\left|\Delta \backslash I_{[w]}\right|-1,2 l([w])+p=r \\ 0, & \text { otherwise. }\end{cases}
$$

Since $E_{s}$ is a local field (Beware of the double-notation!), we conclude by weight arguments (compare also the corresponding argument in loc. cit. Section 5) that the spectral sequence degenerates in $E_{2}$. Thus, we have for $p, r \in \mathbb{N}$,

$$
g r^{p}\left(H_{c}^{r}\left(Y^{\mathrm{ad}}, \Upsilon\right) \otimes_{\mathbb{Z}_{\ell}} \mathbb{Q}_{\ell}\right)=E_{\infty}^{p, r-p}=E_{2}^{p, r-p}=\bigoplus_{[w] \in W^{\mu} / \Gamma_{E_{s}}} E_{2,[w]}^{p, r-p} .
$$


In order to show that the canonical filtration on $E_{\infty}$ splits, we proceed as follows. By unpublished results of Berkovich respectively by Dat ([D], Appendix B.2) we know that the cohomology groups $H_{c}^{r}\left(Y^{\text {ad }}, \Upsilon\right)$ are smooth $J\left(\mathbb{Q}_{p}\right)$-modules. In [O1] (resp. [D] in the split case) the following statement on extensions of generalized Steinberg representations was proved.

Theorem 3.3 ([O1]). Let $r \in \mathbb{N}$ be the $\mathbb{Q}_{p}$-rank of the center of $J$. Let I, $I^{\prime} \subset \Delta$. Then for any $i \in \mathbb{N}$ with $r \geq i \geq\left|I \cup I^{\prime}\right|-\left|I \cap I^{\prime}\right|$,

$$
\operatorname{Ext}_{J\left(\mathbb{Q}_{p}\right)}^{i}\left(v_{P_{I}}^{J}, v_{P_{I^{\prime}}}^{J}\right)=\mathbb{Q}_{\ell}^{\left(\begin{array}{c}
r \\
j
\end{array}\right)}, \quad \text { where } j=i-\left|I \cup I^{\prime}\right|-\left|I \cap I^{\prime}\right| .
$$

For all other $i$ the Ext-group vanishes.

Consider the equation

$$
2 l([w])+\left|\Delta \backslash I_{[w]}\right|-1=r=2 l\left(\left[w^{\prime}\right]\right)+\left|\Delta \backslash I_{\left[w^{\prime}\right]}\right|-1
$$

with $[w],\left[w^{\prime}\right] \in W^{\mu} / \Gamma_{E_{s}}$. If $l([w]) \neq l\left(\left[w^{\prime}\right]\right)$ then $\left|\Delta \backslash I_{[w]}\right|$ and $\left|\Delta \backslash I_{\left[w^{\prime}\right]}\right|$ differ at least by two. Hence $\left|I_{[w]}\right|$ and $\left|I_{\left[w^{\prime}\right]}\right|$ differ at least by two, so that by Theorem 3.3

$$
\operatorname{Ext}_{J\left(\mathbb{Q}_{p}\right)}^{1}\left(v_{P_{[w]}}^{J}, v_{P_{\left[w^{\prime}\right]}}^{J}\right)=0 .
$$

Thus, the expression (7) can be written as

$$
\begin{aligned}
& H^{r}\left(Y^{\mathrm{ad}}, \Upsilon\right) \otimes_{\mathbb{Z}_{\ell}} \mathbb{Q}_{\ell} \cong \bigoplus_{p \in \mathbb{N}} g r^{p}\left(H^{r}\left(Y^{\mathrm{ad}}, \Upsilon\right) \otimes_{\mathbb{Z}_{\ell}} \mathbb{Q}_{\ell}\right) \\
& =\bigoplus_{\substack{[w] \in W^{\mu} / \Gamma_{E_{s}} \\
\left|\Delta \backslash I_{[w]}\right|=1 \\
2 l([w])=r}} i_{P_{[}}^{J} \otimes \operatorname{ind}_{[w]}(-l([w])) \oplus \bigoplus_{\substack{[w] \in W^{\mu} / \Gamma_{E_{s}} \\
\mid \backslash I_{[w]} />1 \\
2 l([w])=r}} i_{J}^{J} \otimes \operatorname{ind}_{[w]}(-l([w])) \\
& \oplus \bigoplus_{\substack{[w] \in W \\
2 l([w])+\left|\Delta \backslash I_{[w]}\right|-1=r \\
p=\left|\Delta \backslash I_{[w]}\right|-1}} v_{P_{\left.I_{[}\right]}}^{J} \otimes \operatorname{ind}_{[w]}(-l([w]))
\end{aligned}
$$

Proof of Theorem 1.1. The proof follows from the formula above together with the long exact sequence (5).

\section{References}

[AS1] G. Alon, E. de Shalit, On the cohomology of Drinfeld's $p$-adic symmetric domain. Israel J. Math. 129 (2002), 1-20. Zbl 1060.14026 MR 1910929

[AS2] G. Alon, E. de Shalit, Cohomology of discrete groups in harmonic cochains on buildings. Israel J. Math. 135 (2003), 355-380. Zbl 1073.14027 MR 1997050 
[B1] V. G. Berkovich, Vanishing cycles for formal schemes. Invent. Math. 115 (1994), 539-571. Zbl 0791.14008 MR 1262943

[B2] V. G. Berkovich, Étale cohomology for $p$-adic analytic spaces. Notes of a talk at Toulouse, June 1994.

[C] W. Casselman, A new non-unitarity argument for $p$-adic representations. J. Fac. Sci. Univ. Tokyo Sect. IA Math. 28 (1981), 907-928. Zbl 0519.22011 MR 0656064

[D] J.-F. Dat, Espaces symétriques de Drinfeld et correspondance de Langlands locale. Ann. Sci. École Norm. Sup. (4) 39 (1) (2006), 1-74. Zbl 05037726 MR 2224658

[dS1] E. de Shalit, Residues on buildings and de Rham cohomology of $p$-adic symmetric domains. Duke Math. J. 106 (1) (2001), 123-191. Zbl 01820775 MR 1810368

[dS2] E. de Shalit, The $p$-adic monodromy-weight conjecture for $p$-adically uniformized varieties. Compositio Math. 141 (1) (2005), 101-120. Zbl 02148431 MR 2099771

[F] L. Fargues, Cohomologie des espaces de modules de groupes $p$-divisibles et correspondances de Langlands locales. Astérisque 291 (2004), 1-199. Zbl 02130511 MR 2074714

[Fo] J.-M. Fontaine, Modules galoisiens, modules filtrés et anneaux de Barsotti-Tate. Astérisque 65 (1979), 3-80. Zbl 0429.14016 MR 0563472

[FR] J.-M. Fontaine, M. Rapoport, L'existence de filtrations faiblement admissibles sur des F-isocristaux. Bull. Soc. Math. France 133 (2005), 73-86. Zbl 1073.14025 MR 2145020

[G] E. Große-Klönne, Frobenius and monodromy operators in rigid analysis, and Drinfel'd's symmetric space. J. Algebraic Geom. 14 (3) (2005), 391-437. Zbl 02204422 MR 2129006

[H1] R. Huber, Étale cohomology of rigid analytic varieties and adic spaces. Aspects Math. E 30, Vieweg, Wiesbaden 1996. Zbl 0868.14010 MR 1734903

[H2] R. Huber, A comparison theorem for $\ell$-adic cohomology. Compositio Math. 112 (1998), 217-235. Zbl 0930.14010 MR 1626021

[IS] A. Iovita, M. Spiess, Logarithmic differential forms on $p$-adic symmetric spaces. Duke Math. J. 110 (2) (2001), 253-278. Zbl 01820855 MR 1865241

[I] T. Ito, Weight-monodromy conjecture for $p$-adically uniformized varieties. Invent. Math. 159 (3) (2005), 607-656. Zbl 02156033 MR 2125735

[J] U. Jannsen, Continuous étale cohomology. Math. Ann. 280 (2) (1988), 207-245. Zbl 0649.14011 MR 0929536

[JP] J. de Jong, M. van der Put, Étale cohomology of rigid analytic spaces. Doc. Math. 1 (1996), 1-56. Zbl 0922.14012 MR 1386046

[K1] R. E. Kottwitz, Isocrystals with additional structure. Compositio Math. 56 (1985), 201-220. Zbl 0597.20038 MR 0809866

[K2] R. E. Kottwitz, Shimura varieties and twisted orbital integrals. Math. Ann. 269 (1984), 287-300. Zbl 0533.14009 MR 0761308

[01] S. Orlik, On extensions of generalized Steinberg representations. J. Algebra 293 (2) (2005), 611-630. Zbl 1080.22008 MR 2173717

[O2] S. Orlik, The cohomology of period domains for reductive groups over local fields. Invent. Math. 162 (3) (2005), 523-549. Zbl 02235497 MR 2198221 
Vol. 82 (2007) The continuous cohomology of period domains over local fields

[R1] M. Rapoport, Two letters to B. Totaro. April 1994.

[R2] M. Rapoport, Non-archimedean period domains. In Proceedings of the International Congress of Mathematicians (Zürich, 1994), Vol. 1, Birkhäuser, Basel 1995, 423-434. Zbl 0874.11046 MR 1403942

[RR] M. Rapoport, M. Richartz, On the classification and specialization of $F$-isocrystals with additional structure. Compositio Math. 103 (1996), 153-181. Zbl 0874.14008 MR 1411570

[RZ] M. Rapoport, T. Zink, Period spaces for p-divisible groups. Ann. of Math. Stud. 141, Princeton University Press, Princeton, NJ, 1996. Zbl 0873.14039 MR 1393439

[S] P. Schneider, The cohomology of local systems on $p$-adically uniformized varieties. Math. Ann. 293 (1992), 623-650. Zbl 0774.14022 MR 1176024

[SS] P. Schneider, U. Stuhler, The cohomology of p-adic symmetric spaces. Invent. Math. 105 (1991), 47-122. Zbl 0751.14016 MR 1109620

[T] B. Totaro, Tensor products in p-adic Hodge theory. Duke Math. J. 83(1) (1996), 79-104. Zbl 0873.14019 MR 1388844

Received June 17, 2005

Sascha Orlik, Universität Leipzig, Mathematisches Institut, Augustusplatz 10/11, 04109 Leipzig, Germany

E-mail: orlik@mathematik.uni-leipzig.de 